УДК 515.124

DOI 10.23671/VNC.2020.1.57537

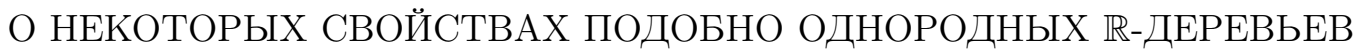

\author{
А. И. Булыгин ${ }^{1}$ \\ ${ }^{1}$ Северный (Арктический) федеральный университет им. М. В. Ломоносова, \\ Россия, 163002, Архангельск, наб. Северной Двины, 17 \\ E-mail: alexey.buligin@gmail.com
}

\begin{abstract}
Аннотация. В работе рассматриваются свойства локально полных подобно однородных неоднородных $\mathbb{R}$-деревьев. Геодезические пространства называются $\mathbb{R}$-деревьями, если любые две точки можно соединить единственной дугой. Рассмотрена общая проблема А. Д. Александрова характеризации метрических пространств. Построены отображения некоторых классов $\mathbb{R}$-деревьев, сохраняющие расстояние один. Для этого используется конструкция, с помощью которой на произвольном метрическом пространстве вводится новая специальная метрика. В терминах этой новой сформулирован признак, необходимый для того, чтобы отображение, сохраняющее расстояние один, было бы изометрией. В рассмотренном случае характеризация А. Д. Александрова не выполняется. Кроме того, в работе исследована граница строго вертикального $\mathbb{R}$-дерева. Доказано, что любая орисфера в строго вертикальном $\mathbb{R}$-дереве является ультраметрическим пространством. Если число ветвления строго вертикального $\mathbb{R}$-дерева не больше континуума, то любая сфера и любая орисфера в $\mathbb{R}$-дереве имеют мощность континуума, а если число ветвления $\mathbb{R}$-дерева больше континуума, то всякая сфера или орисфера будут иметь мощность, равную числу ветвления.
\end{abstract}

Ключевые слова: подобно однородное пространство, вертикальное $\mathbb{R}$-дерево, метрика, орисфера. Mathematical Subject Classification (2000): 54E35, 54E45.

Образец цитирования: Булыгин $A$. И. О некоторых свойствах подобно однородных $\mathbb{R}$-деревьев // Владикавк. мат. журн.-2020.-T. 22, вып. 1.-C. 33-42. DOI: 10.23671/VNC.2020.1.57537.

\section{Введение}

В работе изучаются некоторые свойства подобно однородных неоднородных метрических пространств с внутренней метрикой, в частности $\mathbb{R}$-деревьев. Подобно однородные пространства являются естественным обобщением однородных римановых пространств, геометрия которых хорошо изучена с различных точек зрения, см., например, [1].

Понятие $\mathbb{R}$-дерева, введеное Жаком Титсом в 1977 г. (real-tree, см. [2]), является обобщением понятия симплициального дерева и включается в более общее семейство так называемых $\Lambda$-деревьев [3]. При этом правильно будет отметить, что А. Д. Александров привел две характеризации $\mathbb{R}$-деревьев еще в 1955 г. [4]. Всякое $\mathbb{R}$-дерево является $C A T(\kappa)$-пространством при любом $\kappa \in \mathbb{R}[5]$.

Роль $\mathbb{R}$-деревьев в метрической геометрии и топологии чрезвычайно велика. Они возникают при изучении метрических и топологических пространств в целом ряде ситуаций. М. Громов доказал, что асимптотическим конусом пространства Лобачевского

(c) 2020 Булыгин А. И. 
размерности $n \geqslant 2$ является $\mathbb{R}$-дерево, каждая точка которого является точкой ветвления кратности $2^{\aleph_{0}}$. Конструктивное описание этого $\mathbb{R}$-дерева выполнено А. Дюбиной и И. Полтеровичем в статье [6]. В работах В. Н. Берестовского и К. Плаута (см. [7]), посвященных изучению фундаментальной группы равномерных пространств, вводится понятие универсального $\mathbb{R}$-дерева, которое является аналогом универсального накрывающего пространства, например, для равномерного пространства, не являющегося полулокально односвязным. Показано, что в ряде естественных случаев такое универсальное $\mathbb{R}$-дерево изометрично $\mathbb{R}$-дереву Дюбиной - Полтеровича. Полезный критерий $\mathbb{R}$-дерева в терминах метрических полурешеток получен в статье П. Д. Андреева [8].

Цель настоящей работы состоит в том, чтобы более подробно рассмотреть некоторые свойства симплициальных и вертикальных $\mathbb{R}$-деревьев. Полученные результаты являются продолжением исследований по геометрии подобно однородных $\mathbb{R}$-деревьев, представленных в [9].

Статья организована следующим образом. В первом параграфе приводятся необходимые определения и факты. Отображения $\mathbb{R}$-деревьев, сохраняющие расстояние один, изучаются во втором параграфе. Некоторые свойства строго вертикального $\mathbb{R}$-дерева и возможности их применения приведены в третьем параграфе.

\section{1. Предварительные сведения}

Пусть $(X, d)$ - метрическое пространство. Для числа $\varepsilon>0$ точка $m \in X$ называется $\varepsilon$-серединой между точками $x, y \in X$, если выполнены неравенства

$$
d(x, m), d(y, m)<\frac{1}{2} d(x, y)+\varepsilon .
$$

ОПРЕДЕЛЕНИЕ 1. Метрика $d$ называется внутренней, если для любых точек $x, y \in X$ и любого $\varepsilon>0$ существует $\varepsilon$-середина $m$ между $x$ и $y$, и строго внутренней, если для любых $x, y \in X$ между ними имеется середина $m$ :

$$
d(x, m)=d(y, m)=\frac{1}{2} d(x, y) .
$$

Под отрезком в $X$ с концами $x, y \in X$ понимается образ в $X$ числового отрезка $[a, b] \subset \mathbb{R}$ при изометрическом вложении $i:[a, b] \rightarrow X$, при котором $i(a)=x$ и $i(b)=y$. Пространство $X$ называется геодезическим, если любые две точки $x, y \in X$ можно соединить отрезком. В частности, всякое полное метрическое пространство со строго внутренней метрикой является геодезическим пространством.

Подобием пространства $(X, d)$ с коэффициентом $k>0$ называется биекция $\varphi: X \rightarrow X$, при которой для любых $x, y \in X$ выполнено

$$
d(\varphi(x), \varphi(y))=k \cdot d(x, y) .
$$

В частности, подобие $\varphi$ с коэффициентом $k=1$ является изометрией $X$.

ОПРЕДЕлЕНИЕ 2. Пространство $X$ называется подобно однородным, если для любых точек $x, y \in X$ существует подобие $\varphi$, переводящее $x$ в $\varphi(x)=y$. Если для любых $x, y \in X$ существует изометрия $\varphi$, переводящая $x$ в $y$, пространство $X$ называется однородным. Другими словами, пространство $X$ однородно (соответственно, подобно однородно), если его группа изометрий $\operatorname{Isom}(X)$ (соответственно, группа подобий $\operatorname{Sim}(X))$ действует на $X$ транзитивно.

ОПРЕДЕЛЕНИЕ 3. Пространство $X$ называется локалъно полным, если для любой точки $x \in X$ определено число $r>0$, для которого замкнутый шар $B(x, r)$ полон в метрике $d$. 
Понятно, что в этом случае полными будут и шары $B\left(x, r^{\prime}\right)$ при всех положительных $r^{\prime} \leqslant r$. Точная верхняя грань радиусов $r$, для которых шар $B(x, r)$ полон, называется радиусом полноты в точке $x$. Для радиуса полноты в статье [10] принято обозначение $c(x)$. Если $c\left(x_{0}\right)=+\infty$ хотя бы в одной точке $x_{0} \in X$, то пространство $X$ полное и $c(x)=+\infty$ во всех точках $x \in X$.

Теорема 1 (Берестовский В. Н., [10]). Локально полное подобно однородное пространство $X$ однородно тогда и только тогда, когда оно полно.

В статье рассматриваются исключительно подобно однородные пространства, не являющиеся однородными, поэтому функция $c(x)$ всюду конечна. В этом случае легко доказать, что для любых точек $x, y \in X$ выполнено неравенство

$$
|c(x)-c(y)| \leqslant d(x, y) .
$$

Если $\varphi: X \rightarrow X-$ подобие с коэффициентом $k>0$, то для любой точки $x \in X$ выполнено $c(\varphi(x))=k \cdot c(x)$.

ОПРЕДЕЛЕНИЕ 4. Нетривиальное (содержащее более одной точки) геодезическое пространство $(X, d)$ называется $\mathbb{R}$-деревом, если объединение любых двух отрезков $[x y]$ и $[y z]$ в $X$, пересечение которых есть их общий конец $y$, является вновь отрезком $[x z]$. Иначе говоря, $X$ является $\mathbb{R}$-деревом, если любая сторона произвольного треугольника $\triangle x y z$ в $X$ содержится в объединении двух других сторон: $[x z] \subset[x y] \cup[y z]$.

Еще одно эквивалентное определение $\mathbb{R}$-дерева дано в работе [11] и основано на понятии дуги.

ОПРеДЕЛЕНИЕ 5. Дугой в пространстве $(X, d)$ с концами $x, y \in X$ называется образ числового отрезка $[a, b]$ при вложении (не обязательно изометрическом) $\gamma:[a, b] \rightarrow X$, при котором $\gamma(a)=x$ и $\gamma(b)=y$. Геодезическое пространство $X$ называется $\mathbb{R}$-деревом, если в нем любые две точки $x, y \in X$ соединяются единственной дугой. В этом случае всякая дуга автоматически является отрезком.

ОПредЕлЕниЕ 6. Непрерывная функция $f:[a, b] \rightarrow \mathbb{R}$ называется пилообразной, если:

1. $f$ не является постоянной ни на каком интервале $(c, d) \subset[a, b]$;

2. из того, что $f$ монотонна на интервале $(c, d) \subset[a, b]$ следует, что $\left.f\right|_{(c, d)}-$ линейная функция с угловым коэффициентом \pm 1 .

ОПРЕДЕЛЕНИЕ 7 . Пусть $(Y, d)$ - локально полное подобно однородное неоднородное $\mathbb{R}$-дерево. Оно называется вертикальным, если на каждом отрезке $[x y]$, параметризованном натуральной параметризацией $\gamma:[a, b] \rightarrow Y$ так, что $\gamma(a)=x$ и $\gamma(b)=y$, функция радиуса полноты $c(\gamma(t))$ является пилообразной. Вертикальное $\mathbb{R}$-дерево $(Y, d)$ называется строго вертикальным, если на любом отрезке функция $c(\gamma(t))$ имеет не более одной внутренней точки локального экстремума.

ОПРедЕЛЕНиЕ 8. Числом ветвления $\mathbb{R}$-дерева $X$ в точке $x \in X$ называется кардинальное число $\mathfrak{B}(X)-1$, где $\mathfrak{B}(X)$ - кардинальное число компонент связности множества $X \backslash\{x\}$. Если $\mathfrak{B}(X)=1$, точка $x$ называется терминальной. В этом случае ее число ветвления равно нулю. В случае, когда $\mathbb{R}$-дерево $X$ является однородным или подобно однородным, его число ветвления во всех точках совпадает. В этом случае оно не содержит терминальных точек. Числом ветвления однородного или подобно однородного $\mathbb{R}$-дерева будем называть число ветвления в произвольной его точке.

ПримеР 1. Примером тривиального строго вертикального $\mathbb{R}$-дерева является пространство $\mathbb{R}_{+}=(0 ;+\infty)$ со стандартной метрикой $d(s, t)=|s-t|$. Число ветвления при этом равно единице, а радиус полноты в любой точке $t \in \mathbb{R}_{+}$определяется $c(t)=t$. 
ОПРЕДЕЛЕНИЕ 9 . Пусть $(X, d)$ - строго вертикальное $\mathbb{R}$-дерево, отличное от $\mathbb{R}_{+}$. Будем говорить, что $X$ имеет ветвление кверху (соответственно, ветвление книзу), если всякая точка экстремума радиуса полноты является точкой минимума (соответственно, максимума).

ОПРЕДЕЛЕНИЕ 10. Пусть на метрическом пространстве $(X, d)$ задано отношение частичного порядка $\preceq$, по отношению к которому множество $X$ является $\wedge$-полурешеткой. Это значит, что для любых двух точек $u, v \in X$ определен их инфимум $z=u \wedge v$, т. е. такая точка $z$, что $z \preceq u, z \preceq v$, и для произвольной точки $w \in X$ из $w \preceq u, v$ следует $w \preceq z$. Из антисимметричности отношения $\preceq$ следует, что для любых $u, v \in X$ инфимум $z=u \wedge v$ определен однозначно. Полурешетка $(X, \preceq)$ называется нижне полулинейной, если для любой точки $x \in X$ ее нижний конус

$$
\underline{K}_{x}=\{y \in x \mid y \preceq x\}
$$

линейно упорядочен относительно порядка $\preceq$. Тройка $(X, d, \preceq)$ называется метрической $\wedge$-полурешеткой, если $(X, \preceq)$ является $\wedge$-полурешеткой, и для любых точек $u, v \in X$ выполняется равенство

$$
d(u, v)=d(u, u \wedge v)+d(u \wedge v, v)
$$

\section{2. Отображения $\mathbb{R}$-деревьев, сохраняющие расстояние один}

В 1960-х гг. А. Д. Александров сформулировал общую проблему характеризации метрических пространств:

Найти условия, достаточные для того, чтобы в метрическом пространстве $(M, d)$ выполнялась следующая характеризация изометрий. Всякая биекция $F: M \rightarrow M$, сохраняющая вместе с обратным к ней отображением фиксированное расстояние $r$, есть изометрия.

В этом параграфе показано, что для многих $\mathbb{R}$-деревьев существуют биективные отображения на себя, сохраняющие расстояние один, но не являющиеся изометриями (см. предложение 2 и предложение 3). Для этого используется конструкция, с помощью которой на произвольном метрическом пространстве вводится новая специальная метрика. В терминах этой новой метрики можно сформулировать признак, необходимый для того, чтобы отображение, сохраняющее расстояние один, было бы изометрией.

Пусть $(M, d)$ - произвольное метрическое пространство. Введем на множестве $M$ новую метрику $d_{J}$, которая может принимать конечные значения или значение $\infty$.

ОПРЕДЕЛЕНИЕ 11 . Для точек $x, y \in M$ прыжковый путь длины $n$ между ними определяется как отображение

$$
\gamma:\{0,1, \ldots, n\} \rightarrow M,
$$

при котором $\gamma(0)=x, \gamma(n)=y$ и $d(\gamma(i-1), \gamma(i))=1$ при всех $i \in\{1, \ldots, n\}$. Число $n$ называется длиной прыжкового пути $\gamma$.

Прыжковым расстоянием $d_{J}$ между точками $x, y \in M$ называется минимальная длина прыжкового пути между ними. В частности, считаем $d_{J}(x, x)=0$ для любой точки $x \in M$. Если между точками $x$ и $y$ не существует никакого прыжкового пути, мы полагаем $d_{J}(x, y)=\infty$.

Очевидно, что прыжковое расстояние на $M$ задает метрику, которая может принимать бесконечные значения. 
ОПРЕДЕЛЕНИЕ 12. Пространство $M$ называется прыжково связным, если $d_{J}(x, y)<\infty$ для всех $x, y \in M$. Компонентой прыжковой связности называется максимальное по включению прыжково связное подмножество $\mathscr{A} \subset M$. Для произвольной точки $x \in M$ однозначно определена содержащая ее компонента прыжковой связности $\mathscr{A}_{x}$. Всякое множество, представимое в виде объединения компонент прыжковой связности, называется прыжково инвариантным.

Ключевую роль для следующих построений в этом параграфе играет легко доказываемое

Предложение 1. Пусть множество $M$ допускает разбиение в виде объединения $M=\mathscr{A} \cup \mathscr{B}$ двух непустых непересекающихся прыжково инвариантных множеств $\mathscr{A}$ и $\mathscr{B}$. Предположим, что метрическое пространство $\left(\mathscr{A}, d_{J}\right)$ допускает нетривиальную изометрию $f$ на себя, причем найдутся точки $x \in \mathscr{A}$ и $y \in \mathscr{B}$, для которых $d(x, y) \neq$ $d(f(x), y)$. Тогда метрическое пространство $(M, d)$ допускает биективное отображение $F: M \rightarrow M$, сохраняющее расстояние один, но не являющееся изометрией:

$$
F(x)= \begin{cases}f(x), & x \in \mathscr{A} \\ x, & x \in \mathscr{B} .\end{cases}
$$

ОПРедЕлЕниЕ $13 . \mathbb{R}$-дерево называется симплициальным деревом, если оно обладает структурой одномерного симплициального комплекса. Нульмерные симплексы такого комплекса называются вершинами, а одномерные - ребрами симплициального дерева. Каждому ребру $e=[x y]$ сопоставляется некоторое положительное число $\ell(e)-$ его длина. Метрика симплициального дерева $X$ является внутренней метрикой, при которой расстояния между вершинами $x, y \in X$ равно сумме длин ребер, составляющих метрический отрезок $[x y]$.

Предложение 2. Пусть $X$ - полное симплициальное дерево, для которого все расстояния между вершинами являются рациональными числами и представляются дробями, знаменатели которых равномерно ограничены. Тогда $X$ допускает биективное отображение на себя, сохраняющее расстояние один, не являющееся изометрией.

$\triangleleft$ Пусть $n-$ наименьший общий знаменатель всех дробей, задающих расстояния между вершинами в $X$. Для построения нижеприводимой конструкции, мы можем считать, что все ребра дерева $X$ имеют длину $1 / n$ (ребра большей длины можно разбить на отрезки длины $1 / n)$. Выберем произвольно такие числа $\alpha, \beta$, что $0<\alpha<\beta<\frac{1}{2 n}$. Пусть $\mathscr{A}_{\alpha} \subset X$ (соответственно, $\left.\mathscr{A}_{\beta}\right)$ - множество точек, удаленных от вершин дерева $X$ на расстояние $\alpha$ (соответственно, $\beta$ ). Тогда множества $\mathscr{A}_{\alpha}, \mathscr{A}_{\beta}$ и

$$
\mathscr{A}=\mathscr{A}_{\alpha} \cup \mathscr{A}_{\beta}
$$

прыжково инвариантны, причем метрическое пространство $\left(\mathscr{A}_{\alpha}, d_{J}\right)$ изометрично метрическому пространству $\left(\mathscr{A}_{\beta}, d_{J}\right)$. Прыжковая изометрия $\phi_{J}: \mathscr{A}_{\beta} \rightarrow \mathscr{A}_{\alpha}$ устанавливается по следующему правилу. Для произвольной точки $x \in \mathscr{A}_{\beta}$ однозначно определена ближайшая к ней вершина $z \in X$. Для нее $d(x, z)=\beta$. На отрезке $[x z]$ существует единственная точка $y \in \mathscr{A}_{\alpha}$. Положим $\phi_{J}(x)=y$. Покажем, что отображение $\phi_{J}$ является прыжковой изометрией.

Действительно, всякая точка $y \in \mathscr{A}_{\alpha}$ является внутренней точкой некоторого ребра $[z w]$, и на этом ребре однозначно определена точка $x$, для которой $d(z, x)=\beta$. Она и является прообразом $\phi_{J}^{-1}(y)$. 
Пусть теперь задан прыжковый путь $x=x_{0}, x_{1}, \ldots, x_{n}=p$ из точки $x$ в точку $p \in \mathscr{A}_{\beta}$. Покажем, что путь $y=\phi_{J}\left(x_{0}\right), \phi_{J}\left(x_{1}\right), \ldots, \phi_{J}\left(x_{n}\right)=q$ есть также прыжковый путь из точки $y=\phi_{J}(x)$ в точку $q=\phi_{J}(p)$. Для этого достаточно убедиться, что все расстояния $d_{J}\left(\phi_{J}\left(x_{i-1}\right), \phi_{J}\left(x_{i}\right)\right)$ равны $1 / n$ :

$$
d_{J}\left(\phi_{J}\left(x_{i-1}\right), \phi_{J}\left(x_{i}\right)\right)=1 / n .
$$

Действительно, пусть $z_{i-1}$ - ближайшая вершина к точке $x_{i-1}$, а $z_{i}-$ к точке $x_{i}$. Тогда ровно одна из двух точек $z_{i-1}$ или $z_{i}$ принадлежит отрезку $\left[x_{i-1} x_{i}\right]$. Значит и из точек $\phi_{J}\left(x_{i-1}\right)$ и $\phi_{J}\left(x_{i}\right)$ отрезку $\left[x_{i-1} x_{i}\right]$ принадлежит ровно одна. Легко видеть, что во всех возможных ситуациях равенство (1) выполнено. Следовательно,

$$
d_{J}(p, q) \leqslant d_{J}(x, y) .
$$

Аналогично доказывается обратное неравенство.

Множество $\mathscr{A}=\mathscr{A}_{\alpha} \cup \mathscr{A}_{\beta}$ прыжково инвариантно, а $X$ представляется в виде объединения $\mathscr{A} \cup(X \backslash \mathscr{A})$ двух непустых прыжково инвариантных множеств. Отображение $\phi_{J}$ действует на множестве $\mathscr{A}$, как нетривиальная изометрия. Основываясь на предложении 1 , получаем требуемое отображение $F: X \rightarrow X$ :

$$
F(x)=\left\{\begin{array}{ll}
\phi_{J}(x), & \text { если } x \in \mathscr{A} ; \\
x, & \text { если } x \in(X \backslash \mathscr{A}) .
\end{array} \quad\right.
$$

Можно указать и другую конструкцию отображения, не являющегося изометрией и сохраняющего единичные расстояния.

Пример 2. Рассмотрим произвольное симплициальное дерево $X$, все ребра которого имеют длину $1 / n$.

Отметим теперь ряд свойств вспомогательной функции $\varphi:[0,1 / n] \rightarrow \mathbb{R}, \varphi(t)=$ $t+\frac{\sin (2 \pi n t)}{2 \pi n}$. Она возрастает, $\varphi(t)=t$ при $t \in\left\{0, \frac{1}{2 n}, \frac{1}{n}\right\}, \varphi(t)>(<) t$ при $t \in\left(0, \frac{1}{2 n}\right)$ (соответственно, при $\left.t \in\left(\frac{1}{2 n}, \frac{1}{n}\right)\right), \varphi\left(\frac{1}{n}-t\right)=\frac{1}{n}-\varphi(t)$, см. рисунок 1 .

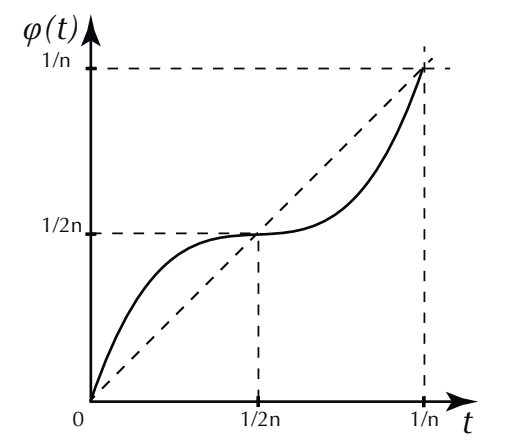

Рис. 1. График функции $t \mapsto \varphi(t)$.

Рассмотрим непрерывное биективное отображение $F: X \rightarrow X$, которое на каждом ребре симплициального дерева действует по правилу

$$
F(\gamma(t))=\gamma(\varphi(t))=\gamma\left(t+\frac{\sin (2 \pi n t)}{2 \pi n}\right)
$$

где $\gamma:\left[0, \frac{1}{n}\right] \rightarrow X-$ натуральная параметризация соответствующего ребра. Нетрудно проверить, что данное отображение сохраняет заданное расстояние и не является изометрией. 
Предложение 3. Пусть $\mathbb{R}$-дерево $X$ допускает нетривиальную изометрию, а множество попарных расстояний между его точками ветвления не более чем счетно. Тогда $X$ допускает биективное отображение на себя, сохраняющее расстояние один и не являющееся изометрией.

$\triangleleft$ Пусть $f: X \rightarrow X-$ нетривиальная изометрия и $P \subset \mathbb{R}_{+}-$счетное множество попарных расстояний между точками ветвления в $X$. Выберем произвольную точку ветвления $x \in X$. Пусть $\mathscr{A}$ - компонента прыжковой связности, содержащая точку $x$. Покажем, что $\mathscr{A}$ не совпадает с $X$.

Действительно, $\mathscr{A}$ представляется как объединение

$$
\mathscr{A}=\bigcup_{k=0}^{\infty} \mathscr{A}_{k},
$$

где

$$
\mathscr{A}_{k}=\left\{y \in X: d_{J}(x, y) \leqslant k\right\} .
$$

Определим отображение $D_{x}: X \rightarrow \mathbb{R}_{+}$равенством $D_{x}(y)=d(x, y)$. Тогда множество значений $\left.D_{x}\right|_{\mathscr{A}}$ не более чем счетно. Действительно, $D_{x}\left(\mathscr{A}_{0}\right)=\{0\}, D_{x}\left(\mathscr{A}_{1}\right)=\{1\}$, и для каждого $k \geqslant 1$ множество значений $D_{x}\left(\mathscr{A}_{k+1}\right)$ получается из $D_{x}\left(\mathscr{A}_{k}\right)$ добавлением некоторого подмножества из множества

$$
\Delta_{k}=\{k+1\} \cup\left\{|1+2 a-b|: a \in P, a \leqslant k, b \in D_{x}\left(\mathscr{A}_{k}\right)\right\} .
$$

Поскольку $\Delta_{k}$ не более чем счетно при всех натуральных $k$, по индукции получаем, что каждое $D_{x}\left(\mathscr{A}_{k}\right)$ не более чем счетно. Следовательно, $D_{x}(\mathscr{A})$ представляется как счетное объединение не более чем счетных множеств, т. е. $D_{x}(\mathscr{A})$ само не более чем счетно. Поэтому оно не может содержать никакого интервала из $\mathbb{R}_{+}$, а в $X$ есть точки, не принадлежащие $\mathscr{A}$.

Множество $\mathscr{A}$ прыжково инвариантно, а $X$ представляется в виде объединения $\mathscr{A} \cup(X \backslash \mathscr{A})$ двух непустых прыжково инвариантных множеств. Отображение $f$ действует хотя бы на одном из этих множеств как нетривиальная изометрия, поскольку $f-$ нетривиальная изометрия на всем $X$. В итоге получаем требуемое отображение $F: X \rightarrow X$.

Следует отметить, что $F$ - биекция, сохраняющая расстояние один, причем этим же свойством обладает обратное отображение $F^{-1}$. Характеризация А. Д. Александрова для метрических пространств в рассмотренном случае не выполняется. Полученный результат согласуется с работами других авторов (см. [12-14]).

\section{3. Граница строго вертикального $\mathbb{R}$-дерева}

ОПРЕДЕЛЕНИЕ 14 . Пусть $(X, d)$ - строго вертикальное $\mathbb{R}$-дерево, имеющее ветвление кверху. Для точек $x, y \in X$ будем говорить, что $x$ предшествует $y$, если на отрезке $[x y]$ минимум радиуса полноты достигается в $x$. В этом случае примем обозначение $x \nearrow y$. Аналогично, пусть $(X, d)$ - строго вертикальное $\mathbb{R}$-дерево с ветвлением книзу. Для точек $x, y \in X$ мы говорим, что $x$ предшествует $y$, если в $x$ достигается максимум радиуса полноты на $[x y]$. В этом случае мы принимаем обозначение $x \searrow y$.

ОПредЕление 15 . Пусть $(X, d)$ - строго вертикальное $\mathbb{R}$-дерево с ветвлением книзу. Определим метрику $d$ на множестве $X$ следующим образом:

a) если $x \nearrow y$ или $y \nearrow x$, то $d(x, y)=\left|\frac{1}{c(x)}-\frac{1}{c(y)}\right|$;

б) если $x$ и $y$ несравнимы в смысле порядка $\nearrow$, то $d(x, y)=d(x, x \wedge y)+d(x \wedge y, y)$. 
Теорема 2 (Андреев П. Д., [8]). Пусть $(X, d)$ - геодезическое пространство. Оно является $\mathbb{R}$-деревом тогда и только тогда, когда на $X$ существует частичный порядок $\preceq$, по отношению к которому тройка $(X, d, \preceq)$ является нижне полулинейной метрической $\wedge$-полурешеткой.

ОПРЕДЕЛЕНИЕ 16. Метрика называется ультраметрикой, если она удовлетворяет усиленному неравенству треугольника $d(x, z) \leqslant \max \{d(x, y), d(y, z)\}$. Пространство $(X, d)$ является ультраметрическим, если метрика $d$, определенная на нем, удовлетворяет данному условию.

В любом строго вертикальном $\mathbb{R}$-дереве $(X, d)$ всякая сфера по отношению к метрике $d$ является ультраметрическим пространством. Если зафиксировать точку $y$, принадлежащую сфере $S(x, d(x, y))$, а центр $x$ устремить к бесконечности по некоторому фиксированному лучу пространства $X$, то сферы $S(x, d(x, y))$ будут сходиться по Хаусдорфу в категории пространств с отмеченной точкой к некоторому множеству. В геометрии Лобачевского предел сфер, полученный таким способом, называется орисферой. В общей геометрии метрических пространств используется аналогия с геометрией Лобачевского, и также предельные множества называются орисферами. Из того, что в $\mathbb{R}$-дереве $(X, d)$ все сферы - это ультраметрические пространства следует, что и все орисферы также являются ультраметрическими пространствами.

Если число ветвления строго вертикального $\mathbb{R}$-дерева не больше континуума, то любая сфера и любая орисфера в $\mathbb{R}$-дереве имеют мощность континуума, а если число ветвления $\mathbb{R}$-дерева больше континуума, то всякая сфера или орисфера будут иметь мощность, равную числу ветвления.

Рассмотрим в строго вертикальном $\mathbb{R}$-дереве с ветвлением книзу сферу $S$, она будет являться ультраметрическим пространством.

Теорема 3. Пусть $(X, d)$ - строго вертикальное $\mathbb{R}$-дерево с ветвлением вниз. Тогда:

1. все множества уровней функции радиуса полноты являются оришарами с центром в единственной бесконечно удаленной точке;

2. обратно, все оришары являются множествами уровня функции $c(x)$;

3. все множества уровня функции $c(x)$ изометричны. Пусть также $\bar{X}$ пополнение пространства $X$, тогда его граница $d X=\bar{X} \backslash X$ изометрична произвольному оришару.

$\triangleleft$ Доказательство теоремы основано на том, что всякое строго вертикальное $\mathbb{R}$-дерево изометрично модельному $\mathbb{R}$-дереву $X_{-}(G)$ для некоторой группы $G[9]$. Для строго вертикальных $\mathbb{R}$-деревьев с ветвлением вниз модельное $\mathbb{R}$-дерево $T$ представляется как множество кусочно-постоянных слева функций вида $f:(a,+\infty) \rightarrow G$, где $a>0, G$ - некоторая группа, причем $\left.f\right|_{(b,+\infty)}=e$ при некотором $b \geqslant a$, где $e-$ единица в $G$. В таком представлении число $a$ равно радиусу полноты функции $f$, как элемента модельного $\mathbb{R}$-дерева. Изометрия множества уровня $c^{(-1)}(s)$ на $c^{(-1)}(t)$ при $t>s$ осуществляется сдвигом вида $f \rightarrow g$, где функция $g:(t ;+\infty) \rightarrow G$ определяется равенством $g(p)=f(p+s-t)$ для всех $p>t$. $\triangleright$

Применение конструкций ультраметрического анализа и пространств данного типа, в приложениях к теории спиновых стекол, динамике макромолекул и генетике, подробно изложены в книге [15].

\section{Литература}

1. Берестовский В. Н., Никоноров Ю. Г. Римановы многообразия и однородные геодезические.Владикавказ: ЮМИ ВНЦ РАН и РСО-А, 2012.-414 c.-(Итоги науки. Юг России. Мат. монография. Вып. 4). 
2. Tits J. A "theorem of Lie-Kolchin" for trees // Contributions to Algebra.-N. Y.: Academic Press, 1977.-P. 377-388.

3. Chiswell I. Introduction to $\Lambda$-trees.-U.K.: Queen Mary \& Westfield College, University of London, 2001. -328 p.

4. Alexandrov A. D. On a Generalization of Riemannian Geometry.-Berlin: Jahresber. Humb. Univ., 1955.

5. Bridson M., Haefliger A. Metric Spaces of Non-Positive Curvature.-Berlin: Springer-Verlag, 1999.643 p.-(Ser. Grundlehren der mathematischen Wissenschaften. Vol. 319.) DOI: 10.1007/978-3-66212494-9.

6. Dyubina A., Polterovich I. Explicit constructions of universal $\mathbb{R}$-trees and asymptotic geometry of hyperbolic spaces // Bull. Lond. Math. Soc.-2001.-Vol. 33, № 6.-P. 727-734. DOI: 10.1112/S002460930100844X.

7. Berestovskii V. N., Plaut C. Covering $\mathbb{R}$-trees, $\mathbb{R}$-free groups and dendrites // Advances in Mathematics.-2010.-Vol. 224, № 5.-P. 1765-1783. DOI: 10.1016/j.aim.2010.01.015.

8. Андреев П. Д. Полулинейные метрические полурешетки на $\mathbb{R}$-деревьях // Изв. вузов. Матем.2007.—№ 6.-C. 3-13. DOI: 10.3103/S1066369X07060011.

9. Andreev P. D., Bulygin A. I. On the Vertical Similarly Homogeneous $\mathbb{R}-$ Trees // Lobachevskii J. Math.2019.--Vol. 40, № 2.-P. 127-139. DOI: 10.1134/S1995080219020033.

10. Берестовский В. Н. Подобно однородные локально полные пространства с внутренней метрикой // Изв. вузов. Математика.-2004.-№ 11.-С. 3-22.

11. Bestvina $M . \mathbb{R}$-trees in topology, geometry and group theory // Handbook of geometric topology / Eds. R. J. Daverman, R. B. Sher.-Amsterdam: Elsevier Science, 2002.-P. 55-91.

12. Александров А. Д. Отображения семейств множеств // Докл. АН СССР.-1970.-Т. 190, № 3.C. 502-505.

13. Богатый С. А., Фролкина О. Д. Изометричность отображений, сохраняющих периметр // Вестн. Московск. ун-та. Сер. 1. Математика. Механика.-2004.-№ 1.-С. 3-11.

14. Berestovskii V. N. Pathologies in Aleksandrov spaces of curvature bounded above // Siber. Adv. Math.2002.-Vol. 12, № 4.-P. 1-18.

15. Козырев С. В. Методы и приложения ультраметрического и $p$-адического анализа: от теории всплесков до биофизики // Совр. пробл. матем.-2008.-T. 12.-C. 3-168. DOI: 10.4213/spm23.

Статвя поступила 15 июля 2019 г.

БулыГИН АЛЕКСЕЙ ИвАНОВИч

Северный (Арктический) федеральный

университет им. М. В. Ломоносова,

аспирант

РОССИЯ, 163002, Архангельск, наб. Северной Двины, 17

E-mail: alexey.buligin@gmail.com

Vladikavkaz Mathematical Journal 2020, Volume 22, Issue 1, P. 32-42

\title{
ABOUT SOME PROPERTIES OF SIMILARLY HOMOGENEOUS R-TREES
}

\author{
Bulygin, A. I. ${ }^{1}$ \\ ${ }^{1}$ Northern (Arctic) Federal University named after M. V. Lomonosov, \\ 17 Severnaya Dvina Emb., Arkhangelsk 163002, Russia \\ E-mail: alexey.buligin@gmail.com
}

\begin{abstract}
In this paper we consider the properties of locally complete similarly homogeneous inhomogeneous $\mathbb{R}$-trees. The geodesic space is called $\mathbb{R}$-tree if any two points may be connected by the unique arc. The general problem of A. D. Alexandrov on the characterization of metric spaces is considered. The distance one preserving mappings are constructed for some classes of $\mathbb{R}$-trees. To do this, we use the construction with the help of which a new special metric is introduced on an arbitrary metric space. In terms of this new metric,
\end{abstract}


a criterion is formulated that is necessary for a so that a distance one preserving mapping to be isometric. In this case, the characterization by A. D. Alexandrov is not fulfilled. Moreover, the boundary of a strictly vertical $\mathbb{R}$-tree is also studied. It is proved that any horosphere in a strictly vertical $\mathbb{R}$-tree is an ultrametric space. If the branch number of a strictly vertical $\mathbb{R}$-tree is not greater than the continuum, then the cardinality of any sphere and any horosphere in the $\mathbb{R}$-tree equals the continuum, and if the branch number of $\mathbb{R}$-tree is larger than the continuum, then the cardinality of any sphere or horosphere equals the number of branches.

Key words: similarly homogeneous space, strictly vertical $\mathbb{R}$-tree, metric, horosphere.

Mathematical Subject Classification (2010): 54E35, 54E45.

For citation: Bulygin, A. I. About Some Properties of Similarly Homogeneous $\mathbb{R}$-Trees, Vladikavkaz Math. J., 2020, vol. 22, no. 1, pp. 32-42 (in Russian). DOI: 10.23671/VNC.2020.1.57537.

\section{References}

1. Berestovskii, V. N. and Nikonorov, Yu. G. Riemannian Manifolds and Homogeneous Geodesics, Vladikavkaz, SMI VSC RAS., 2012, vol. 4, 414 p. (in Russian).

2. Tits, J. A "Theorem of Lie-Kolchin" for Trees, Contributions to Algebra, New York, Academic Press, 1977, pp. 377-388.

3. Chiswell, I. Introduction to $\Lambda$-Trees, U.K., Queen Mary \& Westfield College, University of London, 2001, 328 p.

4. Alexandrov, A. D. On a Generalization of Riemannian Geometry, Berlin, Jahresber. Humb. Univ., 1955.

5. Bridson, M. and Haefliger, A. Metric Spaces of Non-Positive Curvature. Ser. Grundlehren der Mathematischen Wissenschaften, vol. 319, Berlin, Springer-Verlag, 1999, 643 p. DOI: 10.1007/9783-662-12494-9.

6. Dyubina, A. and Polterovich, I. Explicit Constructions of Universal $\mathbb{R}$-Trees and Asymptotic Geometry of Hyperbolic Spaces, Bulletin of the London Mathematical Society, 2001, vol. 33, no. 6, pp. 727-734. DOI: $10.1112 / \mathrm{S} 002460930100844 \mathrm{X}$.

7. Berestovskii, V. N. and Plaut, C. Covering $\mathbb{R}$-Trees, $\mathbb{R}$-Free Groups and Dendrites, Advances in Mathematics, 2010, vol. 224, no. 5, pp. 1765-1783. DOI: 10.1016/j.aim.2010.01.015.

8. Andreev, P. D. Semilinear Metric Semilattices on $\mathbb{R}$-Trees, Russian Mathematics (Izvestiya VUZ. Matematika), 2007, vol. 51, no. 6, pp. 1-10. DOI: 10.3103/S1066369X07060011.

9. Andreev, P. D. and Bulygin, A. I. On the Vertical Similarly Homogeneous $\mathbb{R}$-Trees, Lobachevskii Journal of Mathematics, 2019, vol. 40, no. 2, pp. 127-139. DOI: 10.1134/S1995080219020033.

10. Berestovskii, V. N. Similarly Homogeneous Locally Complete Spaces with an Intrinsic Metric, Russian Mathematics (Izvestiya VUZ. Matematika), 2004, vol. 48, no. 11, pp. 1-19. (in Russian).

11. Bestvina, M. $\mathbb{R}$-Trees in Topology, Geometry and Group Theory, Handbook of Geometric Topology, North-Holland, Elsevier Science, 2002, pp. 55-91.

12. Aleksandrov, A. D. Mappings of Families of Sets, Doklady Akademii Nauk SSSR, 1970, vol. 190, no. 3, p. 502-505 (in Russian).

13. Bogatyj, S. A. and Frolkina, O. D. Isometricity of Mappings Preserving Perimeter, Vestnik Moskovskogo Universiteta. Ser. 1. Matematika Mekhanika, 2004, no. 1, pp. 3-11 (in Russian).

14. Berestovskii, V. N. Pathologies in Aleksandrov Spaces of Curvature Bounded Above, Siberian Advances in Mathematics, 2002, vol. 12, no. 4, pp. 1-18.

15. Kozyrev, S. V. Methods and Applications of Ultrametric and $p$-Adic Analysis: From Wavelet Theory to Biophysics, Proceedings of the Steklov Institute of Mathematics, 2011, vol. 274, suppl. 1, S1-S84. DOI: 10.1134/S0081543811070017.

Received 15 July, 2019

Aleksey I. Bulygin

Northern (Arctic) Federal University named after M. V. Lomonosov,

17 Severnaya Dvina Emb., Arkhangelsk 163002, Russia,

Post-Graduate Student

E-mail: alexey.buligin@gmail.com 